\title{
Minimisation of divergence error in volumetric velocity measurements and implications for turbulence statistics
}

\author{
Charitha M. de Silva · Jimmy Philip · Ivan Marusic
}

Received: date / Accepted: date

\begin{abstract}
Volumetric velocity measurements performed in incompressible fluids are typically hindered by a non zero divergence error due to experimental uncertainties. Here we present a technique to minimise divergence error by employing continuity of mass as a constraint, with minimal change to the measured velocity field. The divergence correction scheme (DCS) is implemented using a constraint based non-linear optimisation. An assessment of DCS is performed using DNS velocity fields with random noise added to emulate experimental uncertainties, together with a Tomographic PIV data set measured in a channel flow facility at a matched Reynolds number to the DNS data $\left(R e_{\tau} \approx 937\right)$. Results indicate that the divergence of the corrected velocity fields are reduced to near zero, and a clear improvement is evident in flow statistics. In particular, significant improvements are observed for statistics computed using spatial gradients such as the velocity gradient tensor, enstrophy and dissipation, where having zero divergence is most important.
\end{abstract}

Keywords Tomo-PIV · Divergence - Wall Turbulence

\section{Introduction}

The pressure and velocity fields of any incompressible fluid are governed by the Navier-Stokes equations and are constrained by the continuity of mass. In direct numerical simulations (DNS) of fluid flow these equations are forced to be satisfied, whereas, in experimentally

C. M. de Silva · J. Philip · I. Marusic

Department of Mechanical Engineering,

University of Melbourne, Melbourne,

VIC, Australia.

E-mail: c.desilva3@pgrad.unimelb.edu.au measured fields the conditions of conservation of momentum and mass are naturally satisfied for any physically realisable flow. However, in most measurements there is a degree of experimental error, which results in the divergence of the velocity field not equaling zero. It is the purpose of this paper to present a technique for correcting such errors in divergence from experiments, when three-dimensional, three-component information is available. In addition, various flow statistics derived from the velocity field is analysed, to understand the implications of correcting for divergence error.

Computing the divergence requires three-dimensional velocity information together with all the associated spatial gradients, which are not easily attainable from experimental techniques. Most measurements over the last century have been performed using single point measurement techniques, particularly in the study of turbulence. These include techniques such as single-wire and multi-wire hotwire anemometry, known for their high temporal response and long time measurement capability [Bruun, 1995]. Recent advancement on these techniques such as those by Park and Wallace [1993], Vukoslavcevic et al. [1991] and Klewicki et al. [1994] to name a few, have enabled us to obtain spatial gradients over a single point using a complex multi-wire setup. However, these measurement probes are not easy to manufacture and implement.

More recently, with the advent of Particle Image Velocimetry (PIV) and its variants, three dimensionalthree component (3D-3C) measurements have become increasingly popular [Adrian and Westerweel, 2011]. The ability to obtain 3D-3C information is advantageous in the study of turbulence since it enables us to obtain velocity gradient information, in particular, quantities such as the components of the velocity gradient tensor (VGT) over a reasonable field of view. Furthermore, we 
are able to quantitatively access the quality of measurements in an incompressible flow by checking how well these measurements adhere to the constraint of zero divergence. Typically a divergence error exists in most 3D-3C measurements, with the magnitude dependent on the quality of the measurement and the flow considered. For instance, large errors are observed in the near-wall region for wall-bounded turbulence due to high velocity gradients present in this region. Certain analyses implicitly require that continuity of mass is satisfied, for example, studies of the invariants of the VGT [Chong et al., 1990, Ooi et al., 1999]. These investigations have been performed primarily by using DNS data, thereby limiting these studies to low Reynolds number flows, which sets aside experimental measurements capable of obtaining a higher Reynolds number flow. In addition, any technique used to determine pressure from a three dimensional velocity field will be erroneous if the divergence is not near zero. Therefore, it would be advantageous to obtain 3D-3C measurements with zero divergence error; or if there is an error, to minimise it.

Here we propose a divergence correction scheme (DCS) which reduces the divergence error to near zero in a typical 3D-3C measurement such that the corrected velocity field is 'not-too-far' from the original experimental data. A quantitative definition of the term 'not-too-far' will be discussed in detail in the $\S 2$ of this paper. It should be noted, that reducing the error in divergence not only improves the accuracy of the VGT, we would also expect an improved measure of the physical properties from the measured flow such as the kinetic energy, enstrophy, dissipation, etc. These improvements are quantified by a comparative study employing DNS velocity fields of del Alamo et al. [2004] from a turbulent channel flow at a Reynolds number of $R e_{\tau}=934$. Noise is added (to be explained in detail below) to the DNS velocity fields prior to applying DCS, after which a comparison is made against the original DNS velocity fields to assess any improvement obtained from DCS. It should be noted that typical 3D-3C measurements such as Tomographic PIV are affected by unbiased random noise, such as electrical noise from the camera [Christensen and Adrian, 2002] and by measurement uncertainties within the velocity field of approximately 0.1-0.2 pixels per vector [Adrian and Westerweel, 2011, Buxton et al., 2011]. In addition, PIV measurements are also affected by several types of biased noise, for example, pixel peak locking which biases displacements to integer values; spatial attenuation due to the interrogation volume size; and also the presence of ghost particles which bias the velocity estimation towards the mean flow. Both bias and unbiased errors in such mea- surements and their impact on DCS are examined in this study. Finally, we perform an experimental validation using Tomographic PIV velocity fields obtained in a channel flow facility with a matched Reynolds number to the DNS data at $R e_{\tau}=937$. The application of DCS to experimental data enables us to assess the practical aspects of the proposed technique.

The later part of this paper is arranged in the following fashion; $\S 2$ provides the general framework of DCS, $\S 3$ and $\S 4$ details the assessment of DCS using DNS and experimental velocity fields, respectively, and finally we summarise the work and conclude in $\S 5$. Throughout this paper $x, y$ and $z$ represent the streamwise, spanwise and wall-normal directions and $U, V$ and $W$ denote respectively the corresponding velocity components. The superscript + refers to normalisation with the viscous inner scale. For example, $l^{+}=l U_{\tau} / \nu$ and $U^{+}=U / U_{\tau}$, where $U_{\tau}$ is the friction velocity and $\nu$ is the kinematic viscosity of the fluid.

\section{Methodology}

Apart from meeting the requirement of continuity and zero divergence, we require that the 'corrected' velocity field, be 'not-too-far' from the experimentally obtained field, such that the corrected velocity field still faithfully represents the original velocity field. To obtain this, we perform a comparison between the corrected and original velocity fields, and try to keep the variation in the averaged kinetic energy (or the $L_{2}$ norm) between the two velocity fields as small as possible. To this end, we propose a divergence correction scheme (DCS) which requires the use of an optimisation algorithm to minimise the objective function (the difference in kinetic energy between the experimental and the corrected velocity fields) given by

$$
\begin{aligned}
F= & \frac{1}{N} \sum_{\text {all points }}\left[\left(U_{c}-U_{\text {exp }}\right)^{2}+\right. \\
& \left.\left(V_{c}-V_{\text {exp }}\right)^{2}+\left(W_{c}-W_{\text {exp }}\right)^{2}\right],
\end{aligned}
$$

with the constraint $\nabla \cdot \mathbf{U}_{\mathbf{c}}=0$; where, $U_{\text {exp }}, V_{\text {exp }}$ and $W_{\text {exp }}$ are the original experimental velocity components, and $N$ denotes the number of points in the velocity field. $U_{c}, V_{c}$ and $W_{c}$ are the corrected/optimised velocity components for which the divergence should be near zero, and the summation is performed across all spatial locations in the velocity field. In the present paper, the divergence of the velocity fields is numerically approximated in the interior of the spatial domain using a second order finite difference scheme given by $\left(\nabla \cdot \mathbf{U}_{\mathbf{c}}=0\right)$ :

$$
\frac{U_{c,(i+1, j, k)}-U_{c,(i-1, j, k)}}{2 \Delta x}+\frac{V_{c,(i, j+1, k)}-V_{c,(i, j-1, k)}}{2 \Delta y}+
$$


$\frac{W_{c,(i, j, k+1)}-W_{c,(i, j, k-1)}}{2 \Delta z}=0$

at each discrete spatial location indicated by $(i, j, k)$, with $\Delta x, \Delta y$ and $\Delta z$ the spacing between them. In a similar fashion, a first order difference scheme is employed at the boundary of the spatial domain.

Equation (2) can be considered as a set of linear equations at each spatial location defined by

$\mathbf{A x}=\mathbf{b}$

where, $\mathbf{x}$ consists of the corrected velocity field components $\left(U_{c}, V_{c}\right.$ and $\left.W_{c}\right)$ to be determined at each spatial location, $\mathbf{b}$ denotes the magnitude of the constraint violation (equal to zero based on equation 2), and $\mathbf{A}$ is a sparse matrix of coefficients obtained from the finite difference schemes to compute gradients at each spatial location. Note that our use of a second order difference scheme is selected to match most PIV studies in the literature, which include Buxton et al. [2011] where a resolution study is performed using DNS and experimental data, and Ganapathisubramani et al. [2005], Worth et al. [2010], to name a few. Nevertheless, higher order or more advanced difference schemes can be implemented instead of the second order central difference scheme used in this study depending on the level of noise associated with the dataset to be corrected and the computational resources available.

The optimisation algorithm described above is implemented in the matrix laboratory computing environment MATLAB, and is solved using the constrained nonlinear multi-variable solver fmincon. We use an exit criteria based on a maximum constraint violation of $1 \times 10^{-7}$ to obtain a comparable divergence error to that observed in the original DNS fields (whereas the typical error in divergence of a $3 \mathrm{D}-3 \mathrm{C}$ experiment is in the order of $1 \times 10^{\circ}$ ). This constraint can be more stringent, but would increase the computational cost depending on the resources available. A maximum tolerance on the objective function (equation 1 ) of $1 \times 10^{-2}$ is used to ensure no major deviation from the initial experimental velocity fields while allowing enough room to perturb the system to satisfy continuity.

It should be noted that, DCS needs to be applied to the complete velocity field of the 3D-3C measurement due to the elliptical nature of the constraint function which is affected by its neighbouring points. However, most 3D-3C measurements usually have partial volumetric velocity fields, such as from Tomo-PIV. Therefore, DCS is limited to the three dimensional volumetric size of the measurement. In addition, edge points in each domain are accounted for by using first order difference schemes (in the present study), which tend to increase the divergence error at these points (even though this could in principle be made higher order). However, this includes only a minimal fraction of the domain, and therefore can reasonably be omitted from the corrected velocity field. Certain measurements may also include boundary conditions such as a no slip condition at a wall present within the velocity field. Although not considered in this study, such conditions can be included in this method (DCS) with relative ease by adding additional constraint equations, enabling us to force the velocity field to meet a certain criteria.

\section{Assessment of DCS using DNS data}

A detailed assessment of the DCS is performed using a direct numerical simulation (DNS) database. The DNS velocity field has a spatial discretisation of Fourier $\times$ Fourier $\times$ Chebyshev with $3072 \times 2304 \times 385$ spatial grid points in the streamwise, spanwise and wall-normal directions respectively. This corresponds to a total computational domain of $8 \pi h \times 3 \pi h \times 2 h$, where $h$ is the half channel height.

For the present purpose, we consider a 'slice' of the full DNS velocity field that approximately corresponds to a typical 3D-3C Tomo-PIV measurement velocity field. Details of the 'slice' considered are summarised in table 1. It should be noted, that 1000 DNS 'slices' are employed to obtain converged first and second order flow statistics. Since the DNS data involves a Chebyshev function to define wall-normal grid spacing, a linear interpolation is employed to obtain the velocity field over a uniform grid in the wall-normal direction making it closer to an experimental dataset. The wall-normal position of the slice $\left(\approx 500<z^{+}<575\right)$, and the fact that a streamwise-spanwise slice is considered here is inconsequential. This is simply selected to minimise the linear interpolation necessary in the wall-normal direction, by minimising the difference between the Chebyshev grid spacing and the uniformly spaced grid. The domain size corresponds to a physical size of 760 and 760 viscous units in the streamwise and spanwise directions respectively, and 75 viscous units in the wallnormal direction.

Table 1 Summary of parameters present in the DNS 'slice'

\begin{tabular}{ll}
\hline Streamwise grid spacing $\left(\Delta x^{+}\right)$ & 7.6 \\
Spanwise grid spacing $\left(\Delta y^{+}\right)$ & 7.6 \\
Wall-normal grid spacing $\left(\Delta z^{+}\right)$ & 7.6 \\
Wall-normal position of slice & $\approx 500<z^{+}<575$ \\
Number of vectors per slice & $100 \times 100 \times 10(x, y, z)$ \\
Size of DNS 'slice' (wall units) & $\approx 760 \times 760 \times 75$ \\
Number of slices used for statistics & 1000 \\
\hline
\end{tabular}




\subsection{Addition of noise to DNS velocity fields}

As detailed previously, typical 3D-3C PIV measurements are affected by both biased and unbiased noise. Here we simulate the effect of unbiased noise by considering two (Case 1 and 2) types of random white noise added to the DNS velocity fields. This type of analysis using random white noise has close similarities to the studies performed by Buxton et al. [2011] on the effects of spatial resolution on kinematic features in turbulence using both experimental and DNS data for PIV experiments. We also consider a case (Case 3 ) where noise is only added to one specific component. This would emulate circumstances where measurement accuracy between the velocity components varies or is biased towards one component. However, it should be noted that several other types of bias error exist in measurements such as Tomo-PIV, which include effects due to spatial averaging across the interrogation volume size and the effect of ghost particles. These types of errors are con- sidered in the latter part of this analysis.

Case 1:

$U_{\mathrm{n}}=U_{\mathrm{o}}+\Gamma_{U}, V_{\mathrm{n}}=V_{\mathrm{o}}+\Gamma_{U}$ and $W_{\mathrm{n}}=W_{\mathrm{o}}+\Gamma_{U}$

where $U_{\mathrm{o}}, V_{\mathrm{o}}$ and $W_{\mathrm{o}}$ correspond to the original DNS velocity field, and $U_{n}, V_{n}$ and $W_{n}$ the 'noisy' velocity field. The 'noisy' velocity field is generated such that $\Gamma_{U}$ is unformly distributed random white noise over a range of $\pm 2 \%$ of $\sqrt{\left\langle U_{\mathrm{o}}^{2}\right\rangle^{+}}$. In other words, noise is added to all three components, with a magnitude based on the dominant velocity component (streamwise). The angle brackets denote volumetric averaging, and the magnitude of the random noise is selected such that the divergence error obtained after the addition of noise (figure $2(\mathrm{~b}))$ is comparable to typical 3D-3C experimental measurements (figure 8(a)).

Case 2:

$U_{\mathrm{n}}=U_{\mathrm{o}}+\Gamma_{U}, V_{\mathrm{n}}=V_{\mathrm{o}}+\Gamma_{V}$, and $W_{\mathrm{n}}=W_{\mathrm{o}}+\Gamma_{W},(5)$
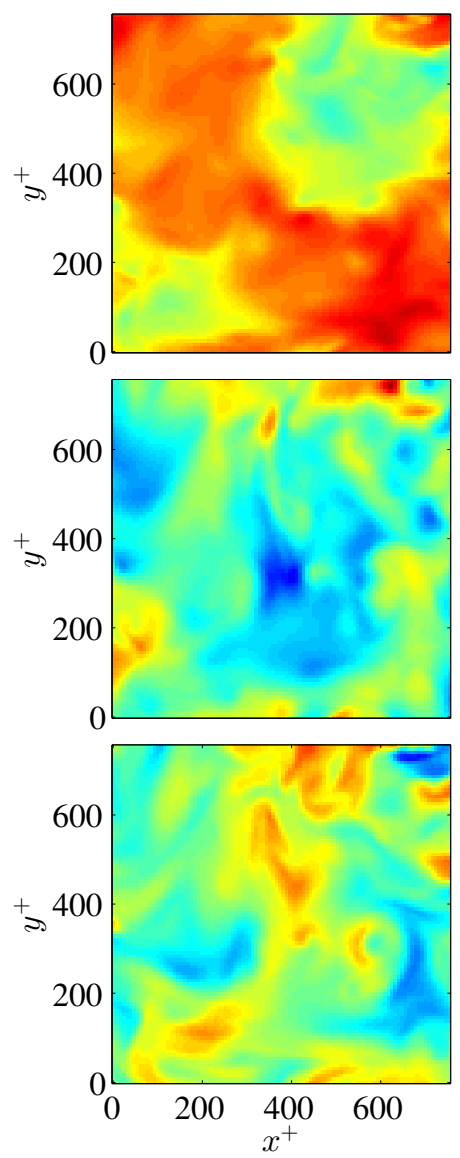

(a) Original DNS
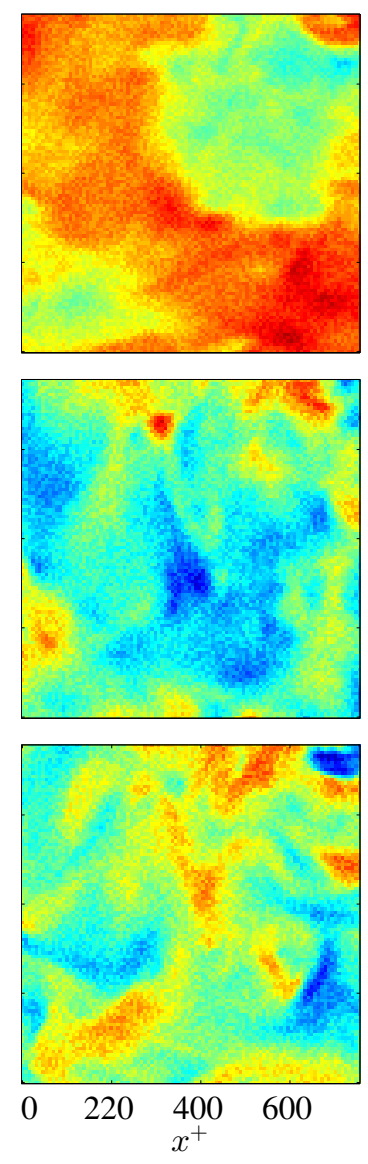

(b) DNS with noise

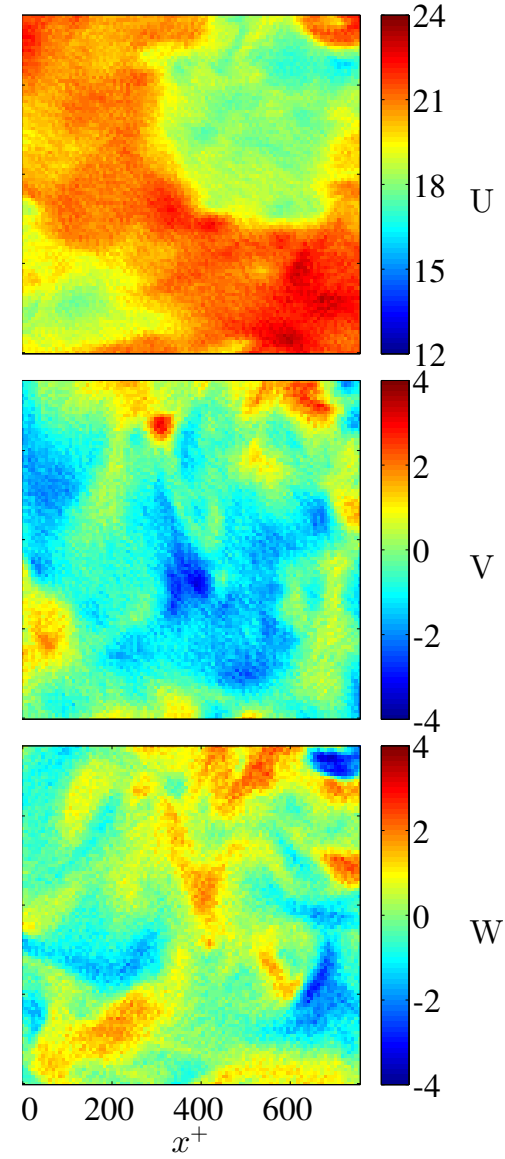

(c) Corrected

Fig. 1 A streamwise-spanwise plane from the DNS data at $z^{+} \approx 540$. The Top, middle and bottom rows correspond to the streamwise, spanwise and wall-normal components, respectively. The left column (a) shows the original DNS velocity field $\left(U_{\mathrm{o}}\right.$, $V_{\mathrm{o}}$ and $\left.W_{\mathrm{o}}\right)$. Similarly, the center column (b) shows the DNS velocity field with noise added based on Case $1\left(U_{n}, V_{n}\right.$ and $\left.W_{n}\right)$, and the right column (c) shows the corrected noisy DNS velocity field $\left(U_{c}, V_{c}\right.$ and $\left.W_{c}\right)$. 


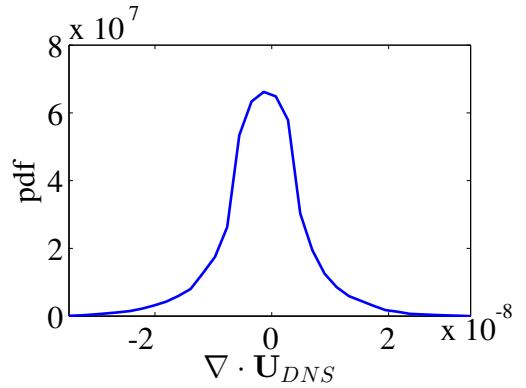

(a) Original DNS

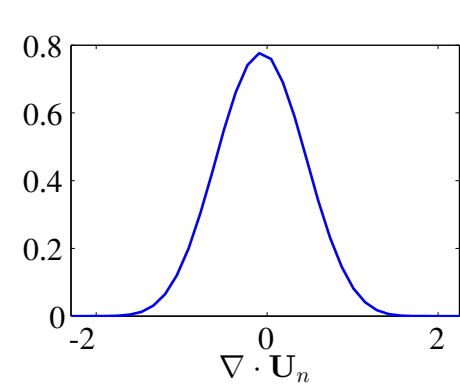

(b) DNS with noise

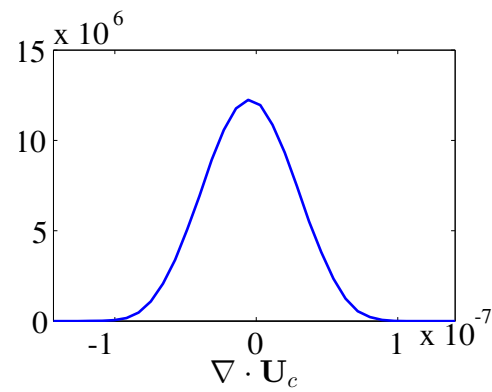

(c) Corrected

Fig. 2 pdf of the divergence obtained from the (a) original DNS velocity field $\left(U_{\mathrm{o}}, V_{\mathrm{o}}\right.$ and $\left.W_{\mathrm{o}}\right)$, (b) DNS velocity field with noise added based on Case $1\left(U_{n}, V_{n}\right.$ and $\left.W_{n}\right)$, and (c) the corrected noisy DNS velocity field $\left(U_{c}, V_{c}\right.$ and $\left.W_{c}\right)$. The abscissa has a range of \pm 5 standard deviations of the divergence in each case, with the original DNS velocity field spanning $\approx \pm 3 \times 10^{-8}$, and the one with noise extending to $\approx \pm 2 \times 10^{0}$.

where $\Gamma_{U}, \Gamma_{V}$ and $\Gamma_{W}$ are $\pm 2 \%$ of $\sqrt{\left\langle U_{\mathrm{o}}^{2}\right\rangle^{+}}, \sqrt{\left\langle V_{\mathrm{o}}^{2}\right\rangle^{+}}$ and $\sqrt{\left\langle W_{0}^{2}\right\rangle^{+}}$respectively.

Case 3:

$U_{\mathrm{n}}=U_{\mathrm{o}}+\Gamma_{U}, V_{\mathrm{n}}=V_{\mathrm{o}}$ and $W_{\mathrm{n}}=W_{\mathrm{o}}$,

where noise is added only to a single velocity component. This enables us to simulate the effect of having a higher reliability in certain velocity components, which is particulary evident in certain types of experiments. For example, in three-component PIV measurements typically the velocity component oriented in the direction of the laser sheet thickness is the least reliable [Adrian and Westerweel, 2011].

Figures 1(a) and 1(b) show streamwise-spanwise planes at $z^{+} \approx 540$ before and after the addition of noise for Case 1 respectively. Since $\Gamma$ is computed using the dominant velocity component $(U)$, all three components show effects of contamination by the random white noise. Figure 2(b) shows the corresponding pdf for the divergence of the noisy data for Case 1 . It should be noted that the pdf obtained is comparable to the pdf from an actual experimental data set (figure 8(a)). The divergence (shown in figure 2(c)) of the corrected velocity field has reduced considerably, close to the range of the original DNS velocity field (shown in figure 2(a)) prior to the application of noise. Furthermore, a comparison between figures $1(\mathrm{~b})$ and 1(c), indicates no significant variation in the three velocity components after correction, which is expected, since the objective function (equation 1) is set up to minimise the variation between the initial velocity field (i.e. noisy velocity field) and the corrected velocity field. We should remind ourselves that DCS is formulated to simply eliminate the divergence error present in the velocity field and not to remove the noise present within a measured velocity field. Nevertheless, it is evident in the analysis to follow that removing the divergence error using DCS does seem to reduce the noise present in the velocity field to some extent.

An alternative technique for removing divergence from Tomo-PIV measurements has been suggested by Clark [2012]. Clark's method shows similar results where no significant improvement is noted in the spatial frequency content, i.e. reduction of the noise. However, reduction of noise as noted previously is not the basis of the correction scheme proposed here. Nevertheless, we note that our results indicate that DCS removes the divergence error while simultaneously improving the velocity field to a certain extent and its corresponding flow statistics when directly compared to DNS velocity fields from both the DNS study and the experimental data (detailed in $\S 4$ ) which is promising. This comparison is possible in the present study since both the DNS velocity fields and the experimental data used in the latter part of the analysis are for a channel flow at the same friction Reynolds number. Furthermore, similar to Clark [2012], a drop in the number of outliers by approximately $10 \%$ is observed in the noisy velocity fields after applying DCS, based on an outlier detection criteria detailed in Westerweel and Scarano [2005].

It should be noted that although we see a considerable reduction in the divergence error, we can also observe the effectiveness of DCS to improve the velocity field by analysing the error of each individual velocity component before and after the application of DCS. Figure 3 shows a pdf of the error defined for the streamwise velocity component as $E_{U_{n}}=U_{\mathrm{n}}-U_{\mathrm{o}}$ and $E_{U_{c}}=U_{\mathrm{c}}-U_{\mathrm{o}}$ before and after the application of DCS respectively. Similar quantities can also be computed for the spanwise and wall-normal velocities. Results indicate that all three components show an improvement in accuracy and a shift towards the original DNS velocity field. In addition, we note that the application of DCS has not produced a bias error in the velocity 


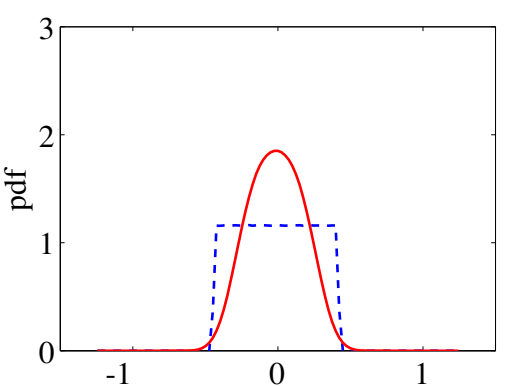

(a) $E_{U^{+}}$

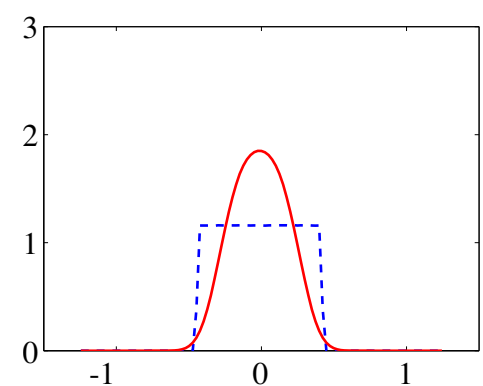

(b) $E_{V^{+}}$

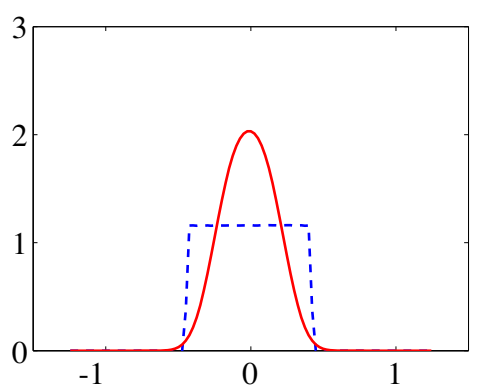

(c) $E_{W^{+}}$

Fig. 3 pdf of the error of the velocity components before and after application of DCS for Case 1. The dashed line indicates the error after the application of randomly distributed white noise, and the solid line indicates the error after application of DCS. (a) Error in the streamwise velocity (e.g $E_{U_{c}}$ for corrected $U$, where $E_{U_{c}}=U_{c}-U_{\mathrm{o}}$ ). Similarly (b) shows spanwise velocity $E_{V}$ and (c), the wall-normal velocity $E_{W}$.
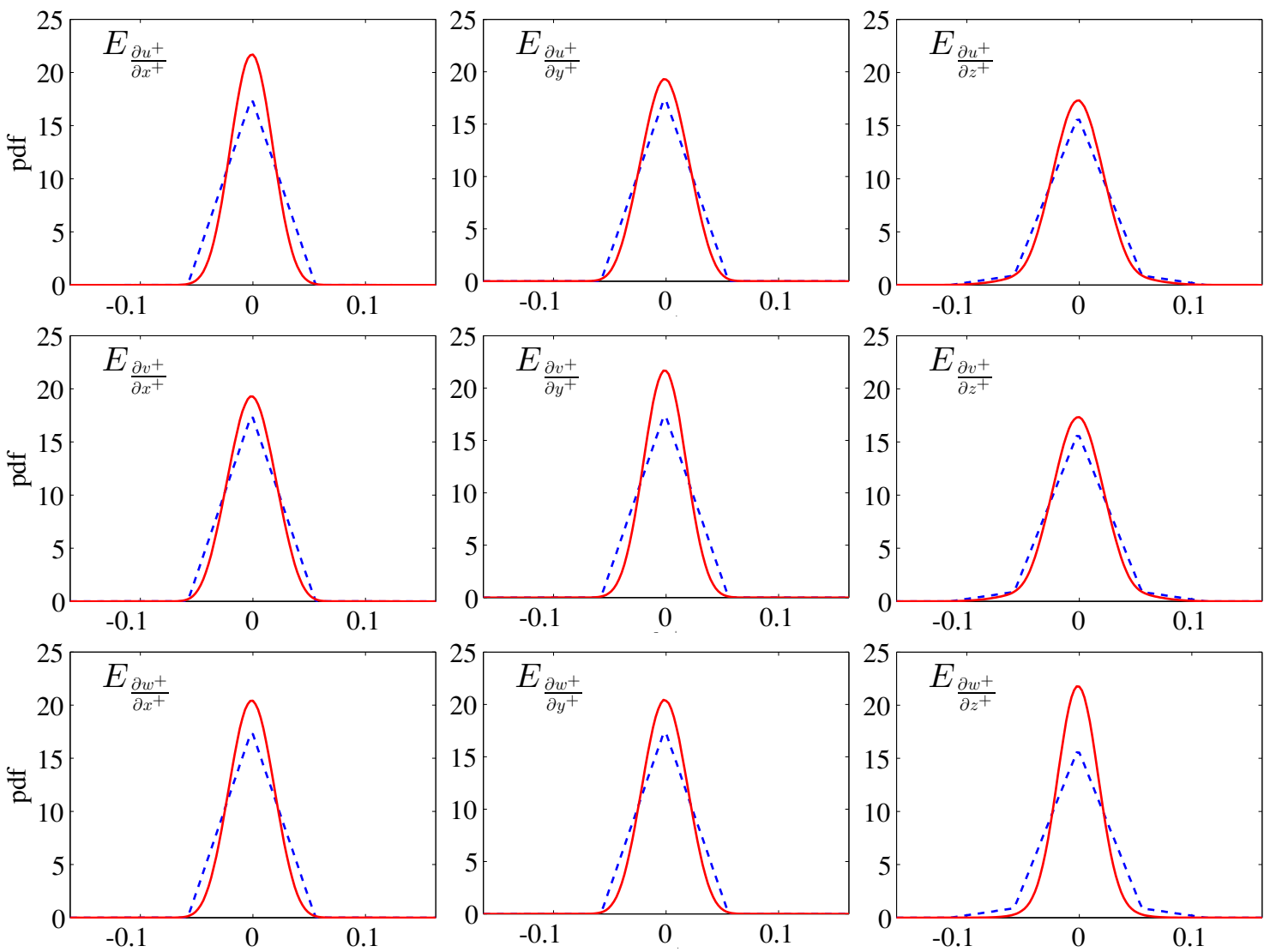

Fig. 4 pdf of the error in spatial gradients before and after application of DCS for Case 1. The dashed line indicates the error after the application of randomly distributed white noise, and the solid line indicates the error after application of DCS.

field within a velocity component (shift in mean error). We note that the simulations are run with uniform grid spacing, where $\Delta x \approx \Delta y \approx \Delta z \approx 7.6$. However it is noted that the grid spacing has an effect on the spatial gradients computed in the DCS technique. Therefore, we would expect increased accuracy if the grid-spacing is reduced for all cases considered in this study. To quantify the associated inaccuracy with each velocity field we compute the normalised variance of the difference for the noisy and corrected velocity fields to the original DNS velocity field. This is computed for the streamwise velocity of the noisy DNS velocity field using

$\mathcal{S}_{U_{\mathrm{n}}}=\frac{\left\langle\left(U_{\mathrm{n}}-U_{\mathrm{o}}\right)^{2}\right\rangle}{\left\langle U_{\mathrm{o}}^{2}\right\rangle}=\frac{\left\langle U_{n}^{2}\right\rangle}{\left\langle U_{\mathrm{o}}^{2}\right\rangle}+1-2 \frac{\left\langle U_{n} U_{\mathrm{o}}\right\rangle}{\left\langle U_{\mathrm{o}}^{2}\right\rangle}$ 
where $\left\langle U_{n} U_{\mathrm{o}}\right\rangle$ is the correlation coefficient (not normalised) between the two signals. In a similar fashion, we can compute equivalent quantities for the spanwise $\left(\mathcal{S}_{V_{\mathrm{n}}}\right)$ and wall-normal $\left(\mathcal{S}_{W_{\mathrm{n}}}\right)$ velocity components, and for the corrected velocity field $\left(\mathcal{S}_{U_{\mathrm{c}}}, \mathcal{S}_{V_{\mathrm{c}}}\right.$ and $\left.\mathcal{S}_{W_{\mathrm{c}}}\right)$. It should be noted that if the signals are perfectly correlated which would typically not be the case, then we can obtain a full picture of the impact or any improvement from DCS by considering only the variances. Therefore, we have considered both the absolute error as described by equation (7), and also the variance of flow statistics before and after the application of DCS (summarised in table 2 for the DNS study and table 5 for the experimental data). We believe this is necessary since equation (7) cannot be applied to the experimental data unlike the study performed with DNS data. Furthermore, a quantitative comparison is performed before and after correcting for divergence error, between flow statistics from the DNS and experimental data (detailed in $\S 4$ ), which is at a matched Reynolds number.

Based on Case 1 for the DNS study, we obtain an improvement (reduction) of approximately $20 \%$ from $\mathcal{S}_{U_{\mathrm{n}}}$ to $\mathcal{S}_{U_{\mathrm{c}}}$ computed using equation (7) in the stream-wise velocity field. Similar improvements of approximately $20 \%$ are obtained for the spanwise and wall-normal velocity components. In addition, figure 4 shows a similar improvement in the pdf of the error associated with each component of the VGT. Cases 2 and 3 also lead to similar results where a reduction in the error is observed. However, these are not shown here for brevity.

As mentioned in $\S 2$, DCS is formulated such that the variations from the initial experimental data to the corrected velocity field (noisy velocity field) is minimal. However, we expect an increase in accuracy in flow statistics, particulary for statistics computed using spatial gradients, since the velocity field now satisfies conti- nuity of mass. To quantitatively assess this, we consider the percentage difference in flow statistics before/after using DCS to the original DNS flow statistics. For example, the percentage difference in the turbulence intensity for the streamwise velocity in the noisy velocity field is defined as,

$\Delta\left\langle u^{2}\right\rangle_{n}^{+}=\frac{\left\langle u^{2}\right\rangle_{n}^{+}-\left\langle u^{2}\right\rangle_{\mathrm{o}}^{+}}{\left\langle u^{2}\right\rangle_{\mathrm{o}}^{+}} \times 100$,

where $\left\langle u^{2}\right\rangle_{\mathrm{o}}^{+}$and $\left\langle u^{2}\right\rangle_{n}^{+}$are the turbulence intensities of the streamwise velocity before and after the addition of noise, respectively. Similarly, $\Delta\left\langle u^{2}\right\rangle_{c}^{+}$can be computed for the corrected velocity field. A comparison of $\Delta\left\langle u^{2}\right\rangle_{c}^{+}$ and $\Delta\left\langle u^{2}\right\rangle_{n}^{+}$enables us to quantify any variation or improvement in the streamwise turbulence intensity after DCS is applied. In a similar fashion, we can compute these quantities for the spanwise and wall-normal velocity components. Comparisons are also drawn for turbulent kinetic energy which is computed from the original DNS velocity field using

$\langle k\rangle_{\mathrm{o}}^{+}=\frac{1}{2}\left[\left\langle u^{2}\right\rangle_{\mathrm{o}}^{+}+\left\langle v^{2}\right\rangle_{\mathrm{o}}^{+}+\left\langle w^{2}\right\rangle_{\mathrm{o}}^{+}\right]$.

as well as, the enstrophy $\left(\langle\boldsymbol{\omega}\rangle_{\mathrm{o}}^{+}=\left[\left\langle\omega_{x}^{2}\right\rangle_{\mathrm{o}}^{+}+\left\langle\omega_{y}^{2}\right\rangle_{\mathrm{o}}^{+}+\right.\right.$ $\left.\left.\left\langle\omega_{z}^{2}\right\rangle_{\mathrm{o}}^{+}\right]\right)$and the dissipation rates $\left(\langle\varepsilon\rangle^{+}=2\left\langle s_{i j}^{+} s_{i j}^{+}\right\rangle\right.$, where $\left.s_{i j}=1 / 2\left[\partial u_{i} / \partial x_{j}+\partial u_{j} / \partial x_{i}\right]\right)$ of the flow.

Table 2 summarises (under the heading for Case 1) the percentage variations for the mean velocity $\langle U\rangle^{+}$, turbulence intensities $\left(\left\langle u^{2}\right\rangle^{+},\left\langle v^{2}\right\rangle^{+}\right.$and $\left.\left\langle w^{2}\right\rangle^{+}\right)$, kinetic energy $\langle k\rangle^{+}$, enstrophy $\left\langle\boldsymbol{\omega}^{2}\right\rangle^{+}$and dissipation $\langle\varepsilon\rangle^{+}$. Column 2 provides the corresponding flow statistics from the original DNS velocity field. Two important conclusions can be drawn from the results presented in table 2 . Firstly, the corrected velocity field is closer to the original DNS velocity field, which indicates an improved representation of the flow field. Secondly, results indicate no significant variation for the mean flow statistics. Therefore, we are still within close proximity to

Table 2 Summary of percentage differences for the mean flow, turbulence intensities, kinetic energy, enstrophy and the dissipation compared to the original DNS statistics prior to and after the application of DCS. $\Delta$ denotes the percentage difference to the original DNS statistics from the noisy and corrected velocity fields.

\begin{tabular}{|c|c|c|c|c|c|c|c|c|}
\hline \multirow{2}{*}{\multicolumn{2}{|c|}{ Original DNS statistics }} & & \multicolumn{2}{|c|}{ Case 1} & \multicolumn{2}{|c|}{ Case 2} & \multicolumn{2}{|c|}{ Case 3} \\
\hline & & & $\begin{array}{l}\text { Noisy } \\
(\%)\end{array}$ & $\begin{array}{l}\text { Corrected } \\
(\%)\end{array}$ & $\begin{array}{l}\text { Noisy } \\
(\%)\end{array}$ & $\begin{array}{l}\text { Corrected } \\
(\%)\end{array}$ & $\begin{array}{l}\text { Noisy } \\
(\%)\end{array}$ & $\begin{array}{c}\text { Corrected } \\
(\%)\end{array}$ \\
\hline \multicolumn{9}{|c|}{$\begin{array}{l}\text { Mean and turbulence } \\
\text { intensity }\end{array}$} \\
\hline$\langle U\rangle^{+}$ & 21.14 & $\Delta\langle U\rangle^{+}$ & 0.0002 & 0.0002 & 0.0002 & 0.0002 & 0.0002 & 0.0002 \\
\hline$\left\langle u^{2}\right\rangle^{+}$ & 1.63 & $\Delta\left\langle u^{2}\right\rangle^{+}$ & 0.93 & 0.64 & 0.95 & 0.51 & 0.94 & 0.49 \\
\hline$\left\langle v^{2}\right\rangle+$ & 0.87 & $\Delta\left\langle v^{2}\right\rangle^{+}$ & 1.77 & 1.22 & 0.03 & 0.06 & 0 & 0.04 \\
\hline$\left\langle w^{2}\right\rangle^{+}$ & 0.66 & $\Delta\left\langle w^{2}\right\rangle^{+}$ & 2.31 & 1.43 & 0.03 & 0.07 & 0 & 0.05 \\
\hline $\begin{array}{l}\text { Kinetic en } \\
\langle k\rangle^{+}\end{array}$ & $\begin{array}{r}\text { gy } \\
1.91 \\
\end{array}$ & $\Delta\langle k\rangle^{+}$ & 1.45 & 0.96 & 0.49 & 0.33 & 0.48 & 0.34 \\
\hline $\begin{array}{l}\text { Enstrophy } \\
\left\langle\boldsymbol{\omega}^{2}\right\rangle^{+}\end{array}$ & 0.0025 & $\Delta\left\langle\boldsymbol{\omega}^{2}\right\rangle^{+}$ & 33.2 & 30.1 & 11.6 & 11.1 & 11.7 & 11.3 \\
\hline $\begin{array}{l}\text { Dissipation } \\
\langle\varepsilon\rangle^{+}\end{array}$ & 0.0025 & $\Delta\langle\varepsilon\rangle^{+}$ & 47.3 & 23.5 & 22.2 & 11.9 & 22.3 & 11.9 \\
\hline
\end{tabular}


the experimental or noisy DNS velocity field used in this study.

A comparison of the turbulence intensities for Case 2 is also summarised in table 2 . Results indicate a similar improvement towards the original DNS velocity field for the streamwise velocity. However, for the spanwise and wall-normal velocity components a deviation away from the original DNS velocity field of $0.03 \%$ and $0.04 \%$ is observed. However, this deviation is minimal, and is caused by the smaller magnitude of the noise added to the spanwise and wall-normal components in Case 2. Furthermore, results indicate an improvement in the $\langle k\rangle^{+},\left\langle\boldsymbol{\omega}^{2}\right\rangle^{+}$and $\langle\varepsilon\rangle^{+}$.

For Case 3, which corresponds to equation (6), we obtain an improvement in flow statistics for the streamwise velocity component comparable to the other cases. Conversely, since noise is not added to the spanwise and wall-normal velocity components, they are slightly perturbed by DCS causing a deviation from the original DNS velocity field. This is indicated in table 2 under Case 3, by a small deviation of $0.04 \%$ and $0.05 \%$ for $\left\langle v^{2}\right\rangle^{+}$and $\left\langle w^{2}\right\rangle^{+}$. It is important to note that the formulation of DCS is such that it consists of one constraint equation and three unknown velocity components at each grid point. Therefore, we would expect it to perturb all three velocity components until the divergence is near zero. However, results from Case 1 indicate that DCS does not add any bias error to the individual components; instead DCS drives the three components towards the original DNS velocity field, which is promising considering this limitation. If we consider Case 3 where noise is only added to the streamwise velocity component, we see that it perturbs the other two components as well, thereby adding error to these components. But as noted previously the deviation of flow statistics from the original DNS velocity field is less than $0.1 \%$ on average.

It should be noted that we use a second order difference scheme in this study primarily due to the reduced computational cost in the optimisation procedure and the lower noise amplification factor associated with a second order difference scheme [Foucaut and Stanislas, 2002]. More recent studies, summarised in Adrian and Westerweel [2011], show that results are less prone to noise when a filter is used on the velocity fields and more advanced difference schemes are employed. However, the use of a spatial filter would cause a certain degree of spatial attenuation, therefore to accommodate this in Case 4 we use both a spatial filter and DCS, thereafter compare to DNS flow statistics at an equivalent resolution (explained in detail in Case 4). This also accounts for the spatial filtering during the measurement process itself (for example, averaging across interroga- tion volumes in Tomo-PIV). Meanwhile, in Cases 1-3 to separate the effect of a spatial filter and DCS we only use DCS on the noisy velocity fields, thereby any noise reduction observed would be caused by DCS alone.

For completeness, the computation of gradients is performed using both a second order central difference scheme and a least squares approach for Case 1 and the experimental data (to be detailed in $\S 4$ ). Both cases indicate comparable improvements in flow statistics after the application of DCS. However, as mentioned previously, due to the increased computational cost associated with using more advanced schemes, and since our primary aim is to demonstrate the effectiveness of the DCS algorithm, the results presented in this paper are obtained using a second order central difference scheme.

\section{Case 4: Biased errors - Spatial Averaging}

3D-3C measurements such as Tomo-PIV are affected by several bias errors. Two main errors include spatial averaging across the interrogation volume and the presence of ghost particles. Both tend to bias the instantaneous displacements towards the mean flow, i.e. they attenuate the displacement fluctuations. Since these effects are present in almost any measurement of this type it is important to consider the influence they may have on the correction scheme proposed in this study. To simulate the effect of bias errors towards the mean flow we apply a three dimensional spatial filter to the DNS velocity field after the addition of noise based on Case 1. A similar approach was used by Buxton et al. [2011] to perform a simulation study using DNS data as described above. Two levels of spatial averaging are considered; one where we use a spatial filter with dimensions $15^{+} \times 15^{+} \times 15^{+}$in all three directions. This is close to the interrogation volume size used for processing the Tomo-PIV data described in $\S 4$, and another where a larger bias error is considered, where the spatial filter size is doubled to $30^{+} \times 30^{+} \times 30^{+}$. It should be noted that the use of a spatial filter does not emulate all the bias errors associated with spatial averaging seen in Tomo-PIV experiments, such as the Tomo-PIV simulations performed by de Silva et al. [2012a], which are computationally intensive due to the nature of processing Tomo-PIV data. Nevertheless, we believe it provides insightful information on the effect of spatial averaging on the correction scheme proposed.

Table 3 summarises the percentage variations for the same flow statistics considered previously at varying spatial resolutions. It should be noted that comparisons are made to filtered DNS statistics which are computed from the DNS velocity fields at an equivalent spatial resolution. This ensures that we minimise the error caused by the discrepancy in spatial resolu- 
Table 3 Summary of percentage differences on the influence of spatial resolution (Case 4) for the mean flow, turbulence intensities, kinetic energy, enstrophy and the dissipation compared to the original DNS statistics prior to and after the application of DCS. $\Delta$ denotes the percentage difference to the original DNS statistics from the noisy and corrected velocity fields.

\begin{tabular}{|c|c|c|c|c|c|c|}
\hline & \multicolumn{2}{|c|}{ DNS Resolution } & \multicolumn{2}{|c|}{$15^{+} \times 15^{+} \times 15^{+}$} & \multicolumn{2}{|c|}{$30^{+} \times 30^{+} \times 30^{+}$} \\
\hline & $\begin{array}{l}\text { Noisy } \\
(\%)\end{array}$ & $\begin{array}{c}\text { Corrected } \\
(\%)\end{array}$ & $\begin{array}{l}\text { Noisy } \\
(\%)\end{array}$ & $\begin{array}{c}\text { Corrected } \\
(\%)\end{array}$ & $\begin{array}{l}\text { Noisy } \\
(\%)\end{array}$ & $\begin{array}{c}\text { Corrected } \\
(\%)\end{array}$ \\
\hline \multicolumn{7}{|c|}{$\begin{array}{l}\text { Mean and turbulence } \\
\text { intensity }\end{array}$} \\
\hline$\Delta\langle U\rangle^{+}$ & 0.0002 & 0.0002 & 0.0002 & 0.0002 & 0.0002 & 0.0002 \\
\hline$\Delta\left\langle u^{2}\right\rangle^{+}$ & 0.93 & 0.64 & 0.79 & 0.50 & 0.31 & 0.26 \\
\hline$\Delta\left\langle v^{2}\right\rangle^{+}$ & 1.77 & 1.22 & 1.62 & 1.35 & 0.28 & 0.23 \\
\hline$\Delta\left\langle w^{2}\right\rangle^{+}$ & 2.31 & 1.43 & 2.29 & 1.61 & 0.32 & 0.24 \\
\hline \multicolumn{7}{|c|}{ Kinetic energy } \\
\hline$\Delta\langle k\rangle^{+}$ & 1.45 & 0.96 & 1.41 & 0.96 & 0.30 & 0.25 \\
\hline \multicolumn{7}{|l|}{ Enstrophy } \\
\hline$\Delta\left\langle\omega^{2}\right\rangle^{+}$ & 33.2 & 30.1 & 28.1 & 27.8 & 23.8 & 24.2 \\
\hline \multicolumn{7}{|c|}{ pation } \\
\hline$\Delta\langle\varepsilon\rangle^{+}$ & 47.4 & 23.5 & 45.8 & 20.5 & 40.4 & 18.7 \\
\hline
\end{tabular}

tion, which could compensate for errors associated with the addition of noise when considering velocity fluctuations. However, we note that because the velocity fields are filtered after the addition of noise as it would be in an experiment, this tends to remove a degree of the noise present prior to applying DCS typically leading to lower errors as observed in table 3 . This effect should not be confused with a compensation of errors due to a variation in spatial resolution as described previously. It should be noted that as the filter size is increased, we note a reduction in the percentage error similar to that observed after the application of DCS. However, we note that DCS is not implemented in a manner to reduce noise from experimental velocity fields, rather to reduce the divergence error to near zero; therefore, we believe the observed reduction in noise after the use of DCS is a bi-product of correcting the divergence error and the fact we have a better representation of the measured flow. In conclusion, we do not directly compare a spatial filter and DCS, rather the two methods seem to be complementing each other and can be used in combination depending on the level of noise in the measurement such as the one discussed in Case 4.

\subsection{Invariants of the velocity gradient tensor (VGT)}

The technique formulated here ensures the continuity of mass in an experimentally measured 3D-3C velocity field. It is important to note that since continuity is computed using spatial gradients, we would expect a significant improvement in flow statistics associated with velocity gradients when DCS is employed. This is observed in flow statistics presented previously in $\S 3$ for the enstrophy and dissipation. To further quantify this effect, we consider the velocity gradient tensor (VGT).
A comprehensive background on the VGT, denoted by $A_{i j}$, and its invariants can be found in Chong et al. [1990]. However, we shall present some salient aspects of the VGT pertaining to the discussion. $A_{i j}$ has the characteristic equation,

$\lambda_{i}^{3}+P_{A} \lambda_{i}^{2}+Q_{A} \lambda_{i}+R_{A}=0$,

where, $\lambda_{i}$ are the eigenvalues of $A_{i j}$ and, $P_{A}, Q_{A}$ and $R_{A}$ the first, second and third tensor invariants, respectively, given by,

$P_{A}=-\operatorname{tr}[\mathbf{A}]$,

$Q_{A}=\frac{1}{2}\left(P^{2}-\operatorname{tr}\left[\mathbf{A}^{2}\right]\right)$, and $R_{A}=-\operatorname{det}[\mathbf{A}]$.

$A_{i j}$ can be split into a symmetric and skew-symmetric components using,

$A_{i j}=S_{i j}+W_{i j}$,

where, $S_{i j}$ is the symmetric rate-of-strain tensor and $W_{i j}$ is the skew-symmetric rate-of-rotation tensor, with,

$S_{i j}=\frac{1}{2}\left(\frac{\partial \dot{x_{i}}}{\partial x_{j}}+\frac{\partial \dot{x_{j}}}{\partial x_{i}}\right)$, and $W_{i j}=\frac{1}{2}\left(\frac{\partial \dot{x_{i}}}{\partial x_{j}}-\frac{\partial \dot{x_{j}}}{\partial x_{i}}\right)$.

It should be noted that in the context of this study the subscripts ' $s$ ' and ' $w$ ' are used to denote the symmetric and anti-symmetric components of the VGT, respectively.

For incompressible flows the first invariant $P_{A}$ is equal to zero and therefore the topology of the flow is only dependent on the second $\left(Q_{A}\right)$ and third $\left(R_{A}\right)$ invariants [Ooi et al., 1999]. However, due to non zero divergence in most volumetric experimental measurements, this is not the case. Therefore, on a two-dimensional $\left(R_{S}, Q_{S}\right)$ plane we observe scatter of experimental data above the null discriminant line $\left(D_{s}\right)$ of $A_{i j}$ given by

$D_{s}=\frac{27}{4} R_{s}^{2}+Q_{s}^{3}$. 
The technique proposed here reduces the error in the divergence to near zero, and therefore, we expect all data points to be below discriminant for the symmetric rate-of-strain tensor. Figures $5(\mathrm{a})$ and $5(\mathrm{~b})$ show a two-dimensional joint-pdf of $R_{A}-Q_{A}$ of the total tensor, and $R_{s}-Q_{s}$ of the symmetric rate-of-strain tensor, respectively, from the DNS data prior to the application of noise. As expected the contour lines indicate that the DNS data satisfy continuity and all points are encapsulated by $D_{A}$ which is shown here by the red full line. In addition, the well documented 'tear-drop' shape is observed on the contour lines in the $R_{A}-Q_{A}$ plane shown
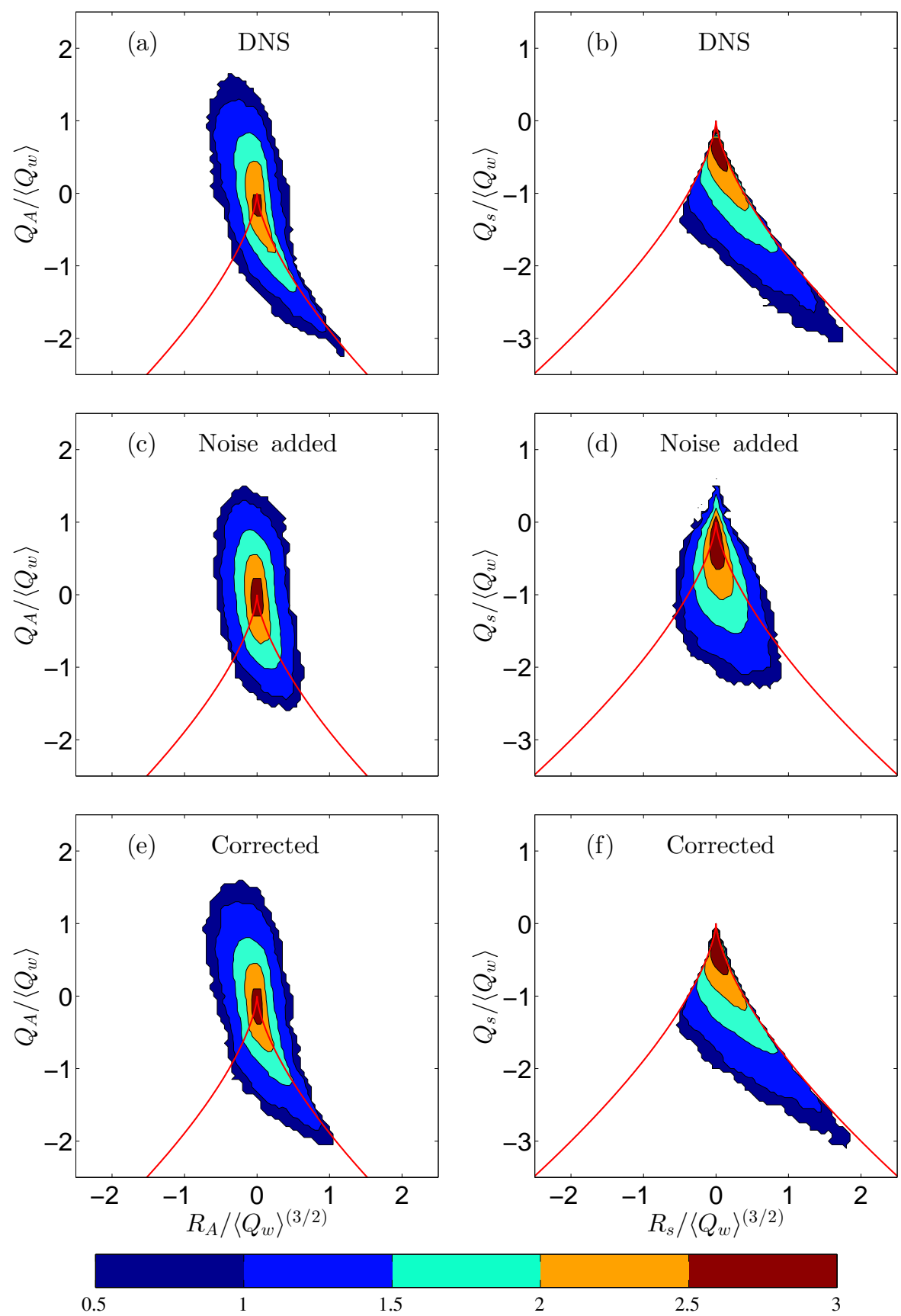

Fig. 5 Joint pdf for $Q_{A}$ vs. $R_{A}$ for the original (a), noisy (c) and the corrected (e) DNS velocity field based on Case 1. The corresponding joint pdf for the symmetric rate-of-strain tensor is shown in figures (b), (d) and (f) respectively. The velocity gradients have been normalised using the ensemble mean based on wall position $z$ which is denoted by the angle brackets. The contour lines are drawn on a logarithmic scale with levels from 0.5 to 2.5 in steps of 0.5 . The solid red line indicates the discriminant of the velocity gradient tensor. 
in figure 5(a) [Ooi et al., 1999]. Once noise is added to the DNS data, a certain proportion of the data now lie outside the bounds of the discriminant due to the non-zero divergence (figure 5(d)). Figure 5(e) and 5(f) show the corresponding joint-pdf after the application of DCS. The contour lines on the $R_{s}-Q_{s}$ plane in figure 5 (f) indicates that the corrected velocity field is free of divergence error and all the data points are below the discriminant $D_{A}$. Furthermore, it can be observed that the 'tear drop' shape which is observed prior to the addition of noise has been recovered to some extent.

\section{Application to experimental data}

The applicability of DCS to 3D-3C measurements is verified by analysing a data set from a Tomo-PIV experiment. The Tomo-PIV data set is obtained at a friction Reynolds number of 937, closely matched to the DNS data used in $\S 3$. This enables us to make a direct comparison between the flow statistics obtained from the experimental and DNS data. The experiments are conducted in the Walter Bassett Aerodynamics Laboratory at the University of Melbourne. Details of the construction of the channel flow facility are provided in Monty [2005]. Figure 6 shows a schematic of the channel flow facility and the experimental setup. The tomographic imaging system consists of four PCO4000 cameras $(4008 \times 2672$ pixels, $2 \mathrm{~Hz})$ equipped with $105 \mathrm{~mm}$ Nikon lenses. The above setup enables us to obtain a reconstructed volume size of $\approx 1.5 h \times 1 h \times 0.2 h$, where $h$ denotes the half-channel height. Further details of the experimental setup can be found in de Silva et al. [2013]. The experimental velocity field is processed using an in-house Tomo-PIV code. Details of the algorithms used for reconstruction and cross-correlation are given in de Silva et al. [2012a]. A pixel to voxel ratio of 1 is maintained in the reconstruction giving a volume size of $4008 \times 2672 \times 534$ voxels. The cross-correlation is performed with an interrogation volume length of $l^{+} \approx 10$, with a corresponding voxel size of $\approx 0.41 \times 0.41 \times 0.41$ viscous units.

Table 4 summarises the parameters of the experimental data set. To reduce the computational time, we have reduced the domain size to one which is comparable to the DNS velocity field used previously. Furthermore, no overlap is used between the interrogation volumes due to the substantial increase in processing time with increased overlap. In addition, the vector spacing is of the order of Kolmogorov length scales associated with the flow based on DNS data at an equivalent Reynolds number [del Alamo et al., 2004]. We note that studies such as Tokgoz et al. [2012] have shown that use of higher overlap may improve the estimates of quantities such as dissipation. We note that the resolution of the experimental velocity field is less than the DNS data, therefore the flow statistics from the experimental data would have higher attenuation compared to the DNS data. Here, the wall-normal location $\left(\approx 375<z^{+}<775\right)$ of the experimental velocity field is selected away from the near-wall region where this attenuation difference is minimal. In addition, the central region of the reconstructed volume is used, which is

Table 4 Summary of parameters used for the experimental data.

\begin{tabular}{ll}
\hline Streamwise grid spacing $\left(\Delta x^{+}\right)$ & $\approx 10$ \\
Spanwise grid spacing $\left(\Delta y^{+}\right)$ & $\approx 10$ \\
Wall-normal grid spacing $\left(\Delta z^{+}\right)$ & $\approx 10$ \\
Number of vectors per slice & $85 \times 40 \times 10(x, z, y)$ \\
Size of velocity field (wall units) & $850 \times 400 \times 100$ \\
Number of slices & 1000 \\
\hline
\end{tabular}

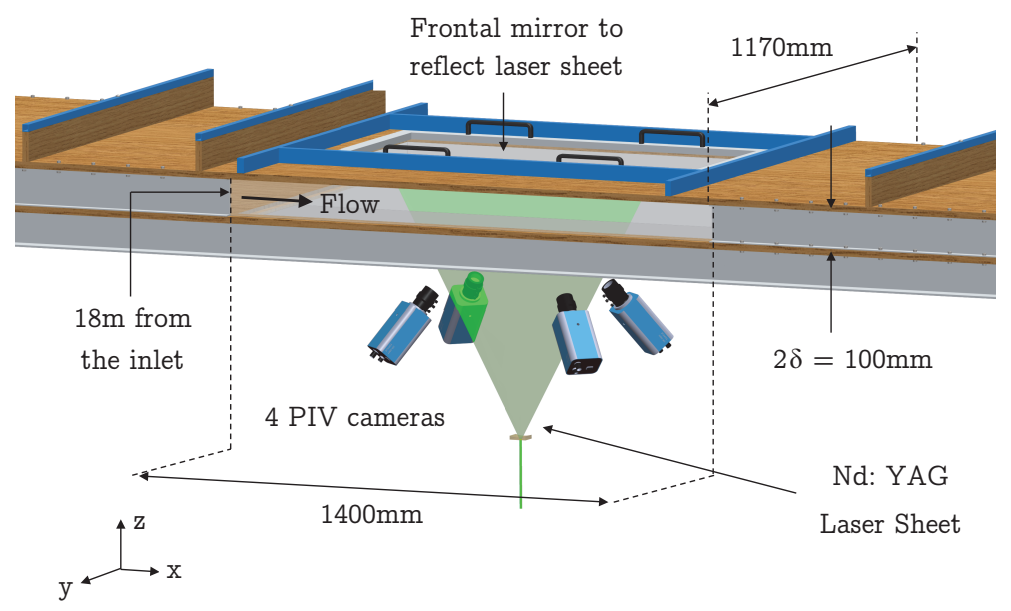

Fig. 6 Experimental setup for Tomo-PIV in channel flow. 
typically where the Tomo-PIV reconstruction provides the best accuracy. Figure 7(a) shows a streamwise wallnormal plane of the experimental data. The corresponding pdf for the divergence error is shown in figure 8(a). The span of the pdf is comparable to that of the pdf obtained after the addition of noise (c.f., figure 2(b)) to the DNS data as mentioned previously for Case 1, as the level of noise is selected to obtain a comparable divergence error. However, we note that the percentage error in flow statistics computed from the experimental data (table 5) is typically higher than that seen in the DNS study (table 2). Therefore, we consider an independent DNS simulation with noise added until a comparable error for $\left\langle u^{2}\right\rangle^{+}$is obtained. Results indicated a similar improvement in flow statistics to that seen in Cases 1-4. However, a comparison of the standard deviation of the divergence error of the noisy DNS velocity fields for this case is approximately $15 \%$ higher than the ex-
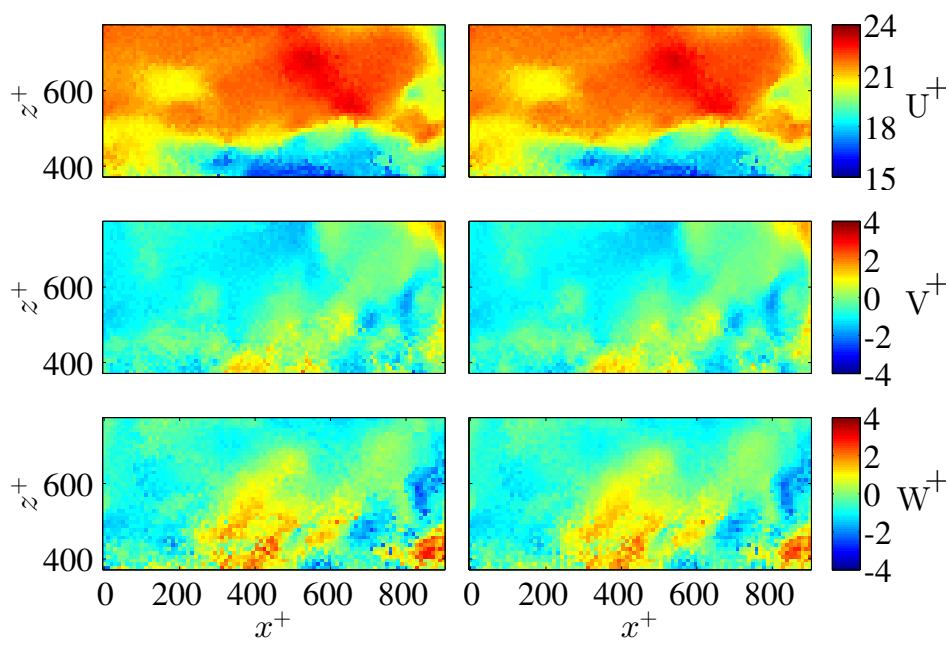

(a) Experimental data perimental data (shown in figure 8(a)). Nevertheless, we still obtain comparable improvements even at this higher level of noise than previously considered in the DNS study.

\subsection{Corrected results for experimental data}

Figure $7(\mathrm{~b})$ shows a streamwise wall-normal plane of the experimental data after the application of DCS. In comparison to the original experimental data shown in figure $7(\mathrm{a})$, no significant variation is observed in all three velocity components. Table 5 summarises a comparison of flow statistics before and after the application of DCS to the experimental data, compared against flow statistics from DNS velocity fields at a matched Reynolds number [del Alamo et al., 2004]. The DNS velocity fields are filtered to match the resolution of the experimental data prior to computing flow statis-

Fig. 7 A streamwise-wall-normal plane from the experimental data in the range $375<z^{+}<775$ for the (a) original experimental velocity field [ (Top) Streamwise $\left(U_{\text {exp }}\right)$, (middle) Spanwise $\left(V_{\exp }\right)$ and (bottom) wall-normal $\left.\left(W_{\exp }\right)\right]$. Similarly, (b) shows the corrected experimental velocity field [ $U_{c}, V_{c}$ and $W_{c}$ ].

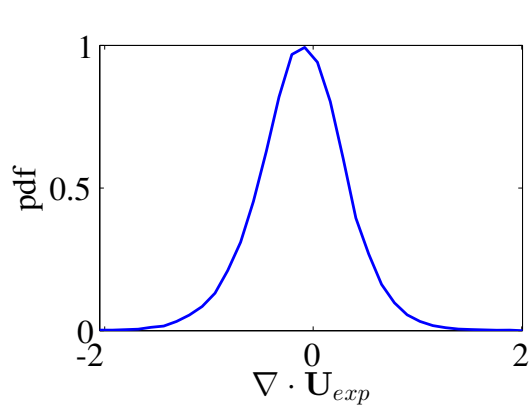

(a) Experimental data

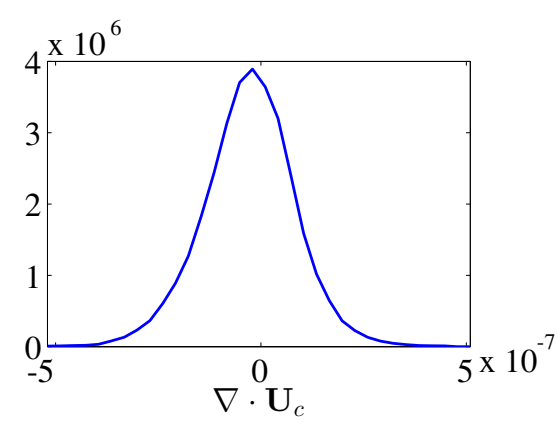

(b) Corrected

Fig. 8 pdf of the divergence obtained from the (a) original experimental velocity field, (b) Corrected experimental velocity field. 
tics, thereby minimising any variation caused by the different spatial resolutions between the experimental and DNS data. A similar approach is performed for Case 4 onwards in the DNS study. It should be noted that since we are not considering the near wall region where the effect of spatial averaging is most significant, we would not expect a significant variation for turbulence intensity statistics. However, this effect is prominent when comparing statistics such as $\left\langle\boldsymbol{\omega}^{2}\right\rangle^{+}$or $\langle\varepsilon\rangle^{+}$ [de Silva et al., 2012b] even away from the wall, since it is computed using spatial gradients. A comparison of the mean velocity and turbulence intensity statistics shown in table 5 indicates that an improvement is observed towards the DNS statistics, similar to results seen by using noisy DNS velocity fields. Moreover, when considering flow statistics obtained from spatial gradients such as dissipation, a considerable improvement is observed. In summary, similar to the study performed with noisy DNS velocity fields DCS has reduced the divergence of the experimental velocity field to near zero (figure 8(b)) while also improving the flow statistics.

The matched Reynolds number between the experimental and DNS data enables us to make a direct com-
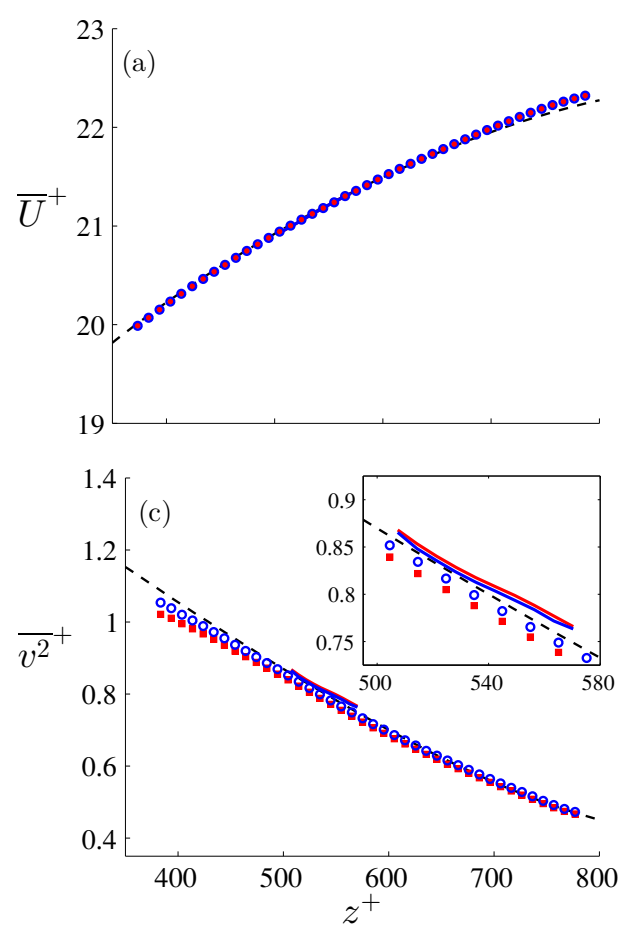

Table 5 Summary of percentage differences for the experimental data for the mean flow, turbulence intensity, kinetic energy, enstrophy and the dissipation compared to filtered DNS statistics obtained at a matched Reynolds number [del Alamo et al., 2004] prior to and after the application of DCS. $\Delta$ denotes the percentage difference to the matched DNS statistics from the original and corrected experimental velocity fields.

\begin{tabular}{|c|c|c|c|c|}
\hline \multirow{2}{*}{\multicolumn{2}{|c|}{ Filtered DNS statistics }} & & \multicolumn{2}{|c|}{ Experimental data } \\
\hline & & & $\begin{array}{l}\text { Original } \\
(\%)\end{array}$ & $\begin{array}{c}\text { Corrected } \\
(\%)\end{array}$ \\
\hline \multicolumn{5}{|c|}{$\begin{array}{l}\text { Mean and turbulence } \\
\text { intensity }\end{array}$} \\
\hline$\langle U\rangle^{+}$ & 21.31 & $\Delta\langle U\rangle^{+}$ & 0.172 & 0.177 \\
\hline$\left\langle u^{2}\right\rangle+$ & 1.52 & $\Delta\left\langle u^{2}\right\rangle+$ & 2.90 & 2.78 \\
\hline$\left\langle v^{2}\right\rangle+$ & 0.75 & $\Delta\left\langle v^{2}\right\rangle+$ & 3.95 & 2.02 \\
\hline$\left\langle w^{2}\right\rangle^{+}$ & 0.62 & $\Delta\left\langle w^{2}\right\rangle^{+}$ & 6.29 & 4.31 \\
\hline \multicolumn{5}{|c|}{ Kinetic energy } \\
\hline$\langle k\rangle^{+}$ & 2.60 & $\Delta\langle k\rangle^{+}$ & 6.05 & 5.18 \\
\hline $\begin{array}{l}\text { Enstrophy } \\
\left\langle\omega^{2}\right\rangle^{+}\end{array}$ & 0.0022 & $\Delta\left\langle\omega^{2}\right\rangle^{+}$ & 12.07 & 11.98 \\
\hline $\begin{array}{l}\text { Dissipation } \\
\langle\varepsilon\rangle^{+}\end{array}$ & 0.0020 & $\Delta\langle\varepsilon\rangle^{+}$ & 38.20 & 29.10 \\
\hline
\end{tabular}

parison between profiles of the flow statistics. Figure 9 shows the mean velocity $U^{+}$, and the turbulence inten-
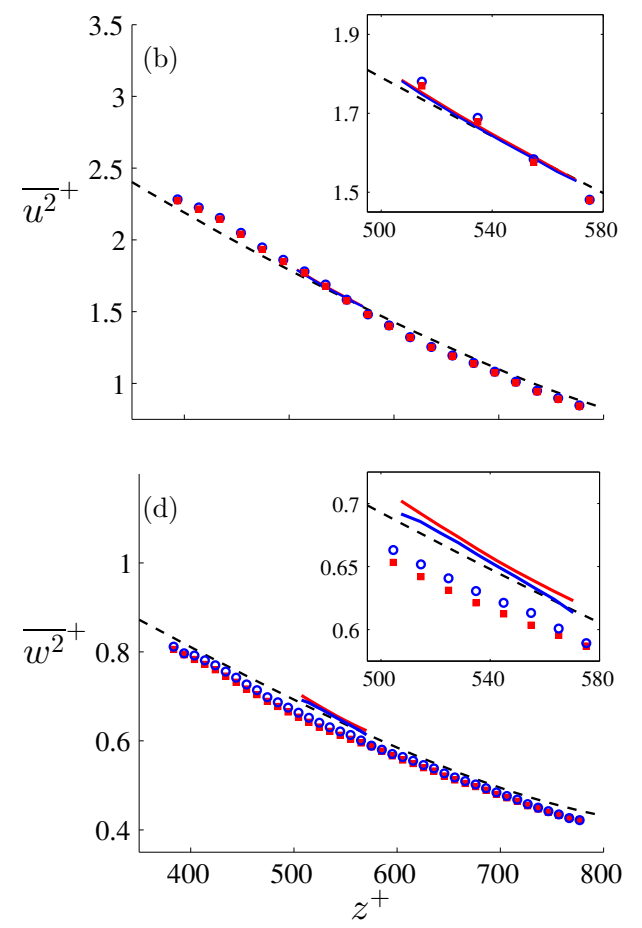

- - - DNS —-DNS with noise —Corrected (DNS with noise) $\quad$ - Exp. data $\quad \circ$ Corrected (Exp. data)

Fig. 9 Comparison of mean velocity(a) and turbulence intensity(b-d) flow statistics for Case 1 from the DNS study as detailed in $\S 3$, together, with flow statistics from the experimental data before and after the application of DCS. Here, a direct comparison can be made since both the DNS and experimental data are at matched Reynolds numbers. The inserts (b-d) includes an expanded view of the $z^{+}$range from the DNS study. Filtered DNS statistics are not included in this plot as minimal attenuation is observed at the spatial resolution of the experimental data, as we are away from the near wall region. 
sities $\left({\overline{u^{2}}}^{+},{\overline{v^{2}}}^{+}\right.$and ${\overline{w^{2}}}^{+})$for the original DNS data (dashed lines), DNS with noise (full, red lines), corrected noisy DNS (full, blue lines), original experimental data (full red symbols) and the corrected experimental data (empty blue symbols). The profiles are plotted against wall-normal location $(z)$ which is a common practice in wall turbulence. As expected, no variation in the mean streamwise velocity is observed, therefore we can conclude that the application of DCS has not modified, or added any bias, to the mean flow of the velocity field. Furthermore, although not easily visible on figure 9 (b), a shift in ${\overline{u^{2}}}^{+}$towards the original DNS is observed, in accordance with the results presented in tables 2 and 5. A comparison of ${\overline{v^{2}}}^{+}$(figure 9(c)) and ${\overline{w^{2}}}^{+}$(figure 9(d)) shows a visible improvement in both the noisy DNS data and the experimental data, since the magnitude of the uncertainty associated with these velocity components are higher. It should be noted, that the statistics $v^{2+}$ and $w^{2+}$ for the experimental data are initially below the original DNS statistics prior to the application of DCS. Once corrected, we observe a shift upwards, towards the statistics obtained from the original DNS data. This implies that DCS is not simply acting as a noise reducing 'filter', which always 'reduces' the turbulence intensity. Instead the suggestion is that DCS acts as a corrective mechanism. Therefore, we believe that correcting for the divergence error provides a better representation of the flow field, which ex- plains the improvement observed in both experimental and the noisy DNS data. A comparison of dissipation rates $\left(\varepsilon^{+}\right)$which is shown in figure 10, indicates that the Tomo-PIV measurement provides a reasonable estimate for $\varepsilon^{+}$, considering that nine spatial gradients are necessary to compute $\varepsilon^{+}$. However, a considerable improvement in both the noisy DNS and experimental data are obtained after the application of DCS. Furthermore, a qualitative improvement in the trend of $\varepsilon^{+}$ at varying $z$ is observed in the experimental data after using DCS (blue, empty symbols).

One should note that obtaining the dissipation rates requires the use of a 3D-3C measurement technique, which has only recently been applied to wall-bounded flows. Typically $\varepsilon^{+}$is approximated based on local isotropy, where its surrogate, $\varepsilon_{i s o}$ is computed using

$\varepsilon_{i s o}=15 \nu \overline{\left(\frac{\partial u}{\partial x}\right)^{2}}$.

This approximation is commonly used for single point measurements such as hot-wire anemometry data where Taylors hypothesis is used to project a time-series of velocity to a spatially resolved series of data. Figure 10 also compares $\varepsilon_{i s o}^{+}$and $\varepsilon^{+}$from the original DNS data, in dashed dotted and dashed lines, respectively. The approximation seems to suffer in the near wall region due to the large inhomogeneity in the wall-normal direction. As $z$ increases the approximation improves in accuracy as expected since the inhomogeneity reduces.

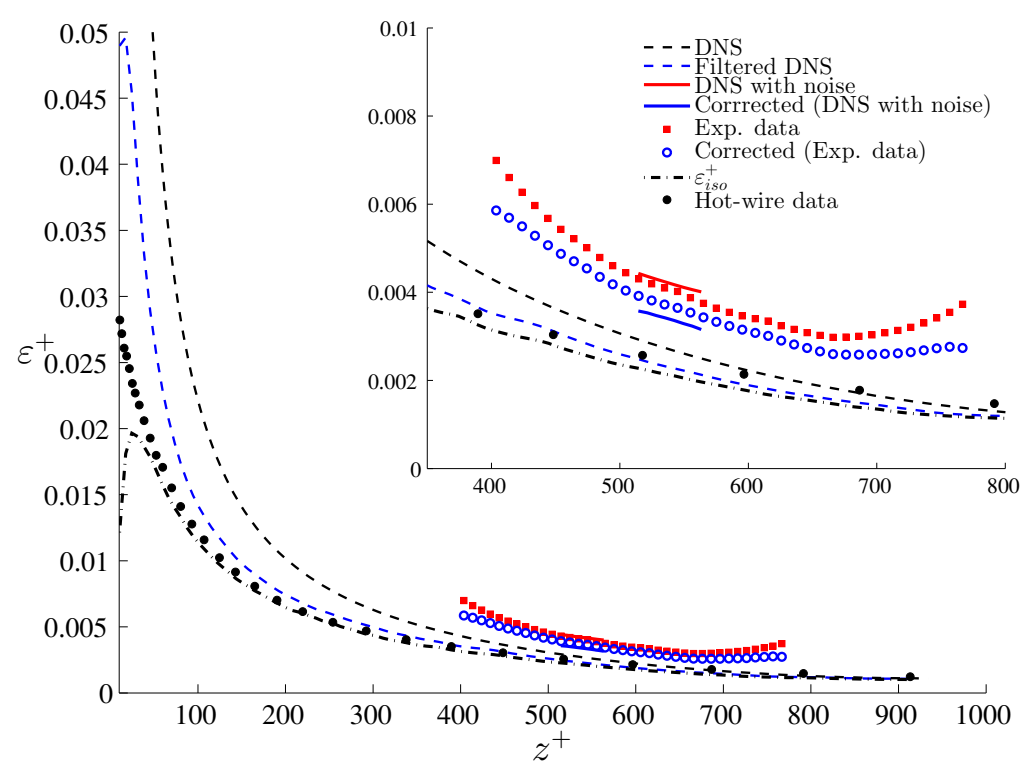

Fig. 10 Comparison of dissipation $\left(\varepsilon^{+}\right)$for Case 1 from the DNS study as detailed in $\S 3$, together with flow statistics from the experimental data before and after the application of DCS. The dash-dotted black line is obtained using the approximation $\left(\varepsilon_{i s o}\right)$ based on isotropic turbulence from the original DNS velocity fields, where $\varepsilon_{i s o}$ is calculated using equation (16). Similarly the black dots are obtained from a hot-wire anemometry measurement by $\mathrm{Ng}$ [2011] at a matched Reynolds number. The insert includes an expanded view of the $z^{+}$range from the Tomo-PIV experiment. Filtered DNS statistics are also included at a matched resolution to the experimental data to visualise the attenuation observed for $\varepsilon^{+}$well away from the wall. 
It should be noted that, $\varepsilon_{i s o}$ is computed directly from the DNS velocity fields. Furthermore, we also present a measurement from hot-wire anemometry (in black dots) at a matched Reynolds number in the same facility $[\mathrm{Ng}$, 2011] for comparison. Figure 10 shows that the hot-wire measurement has a similar trend to $\varepsilon_{i s o}$ from the DNS data, since it is computed using the same approximation (equation 16). The results indicate that the hotwire measurement is in close proximity to the original DNS data in the region $375<z^{+}<775$ and sometimes even better than the Tomo-PIV measurement. We would typically expect the $3 \mathrm{D}-3 \mathrm{C}$ measurement to perform better since we have all spatial gradients, which enables us to directly compute $\varepsilon^{+}$. However, 3D-3C measurements are affected by uncertainties and errors which add measurement noise [Elsinga et al., 2006]; this is observed in our results where $\varepsilon^{+}$is over-estimated for both the Tomo-PIV and the noisy DNS data. In addi-
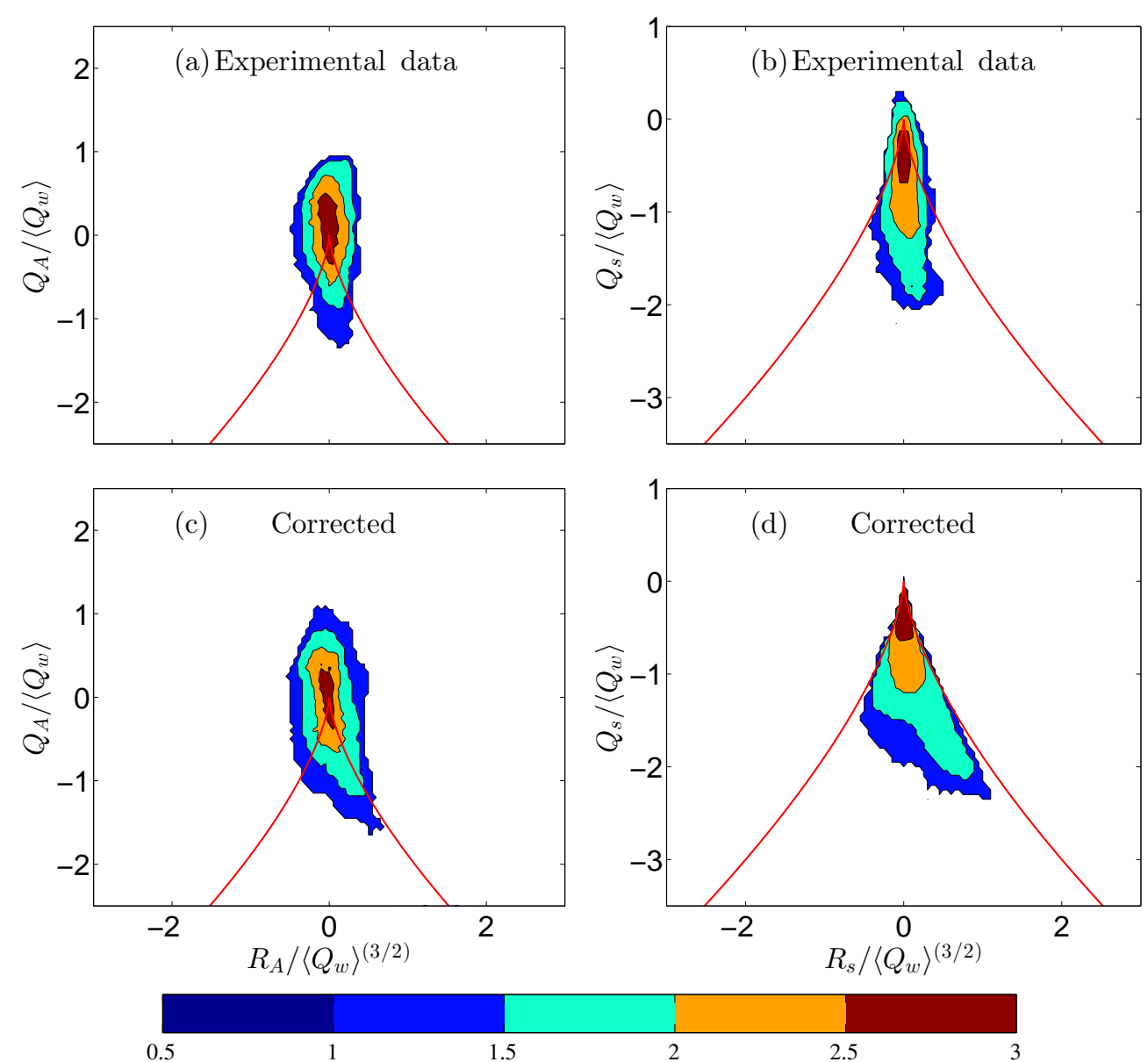

Fig. 11 Joint pdf for $Q_{A}$ vs. $R_{A}$ for the original (a) and the corrected (c) velocity field from the experimental data. The corresponding joint pdf for the symmetric rate-of-strain tensor is shown in figures (b) and (d) respectively. The velocity gradients have been normalised using the ensemble mean based on wall position $z$ which is denoted by the angle brackets. The contour lines are drawn on a logarithmic scale with levels from 1.25 to 2.5 in steps of 0.25 . The solid red line indicates the discriminant of the velocity gradient tensor.

tion, the resolutions between the two techniques are not matched. Here the spanwise averaging in the hot-wire measurement is 22 wall units, compared to an averaging of 10 wall units in all three spatial directions for the Tomo-PIV. Therefore, it is difficult to conclusively compare $\varepsilon^{+}$between hot-wire anemometry and TomoPIV, instead we can gauge how well the approximation based on isotropic turbulence works.

4.2 Invariants of the velocity gradient tensor (VGT) from experimental data.

Similar to the analysis performed in $\S 3.2$ we can compute the invariants of the velocity gradient tensor using equations (11) and (12). Figure 11 shows a twodimensional joint-pdf of $R_{A}-Q_{A}$ of the total tensor and $R_{s}-Q_{s}$ of the symmetric rate-of-strain tensor, before and after the application of DCS to the experimen- 
tal data. The contour lines on the $R_{s}-Q_{s}$ plane (figure 11(b)) indicate that the original experimental data are not divergence free and a large amount of data points lie above the discriminant $D_{A}$ (similar to figure $5(\mathrm{~d}$ ) for the noisy DNS velocity fields). Furthermore, the contour lines in the $R_{A}-Q_{A}$ plane for the original experimental data (figure 11(a)) shows a more 'elliptical shape' in comparison to the 'teardrop' shape obtained from the original DNS velocity field (figure 5(a)). We believe this is primarily caused by the uncertainty and the noise associated with the experimental velocity fields in comparison to DNS velocity fields. However, after the application of DCS the contour lines on the $R_{A}-Q_{A}$ plane are more representative of the typical 'tear-drop' shape but still somewhat far from the ideal expected shape. Having noted that, we should concentrate on the contour lines of the $R_{s}-Q_{s}$ plane (figure 11(d)), which show that the experimental velocity field has near zero divergence error, as indicated by the majority of the data being below the discriminant $D_{s}$, in addition to a comparable shape to that obtained in the DNS study.

\section{Summary and conclusions}

A technique to reduce the divergence error in 3D-3C measurements such as Tomo-PIV has been detailed. This technique is formulated to minimise deviation from the original experimental data while satisfying continuity of mass. An assessment using DNS velocity fields by adding random white noise indicates that the technique reduces the divergence to near zero, with both biased and unbiased noise between the three velocity components. Results indicate an improvement in each velocity component towards the original DNS for noise levels within a range of $\pm 2 \%$ of $\sqrt{\left\langle U_{0}^{2}\right\rangle^{+}}$(determined by comparison to typical measurement noise in 3D-3C experiments). An improvement is also observed in the analysis of the invariants of the velocity gradient tensor (VGT) after the application of the divergence correction scheme (DCS). Furthermore, flow statistics for the mean velocity, turbulence intensity, kinetic energy, enstrophy and dissipation, before and after the application of DCS shows a shift towards the original DNS flow statistics. This further verifies that we obtain a more accurate representation of the flow field after DCS is employed. Figures 12 shows the standard deviations of the divergence corresponding to the three cases of noise addition considered here (i.e, where noise is added to $U, V$ and $W$ based on $U$; on $U, V$ and $W$; and where noise is only added to $U$ ). Results indicate that in all cases considered, the divergence is near zero $\left(\approx 10^{-8}\right)$ after application of DCS.

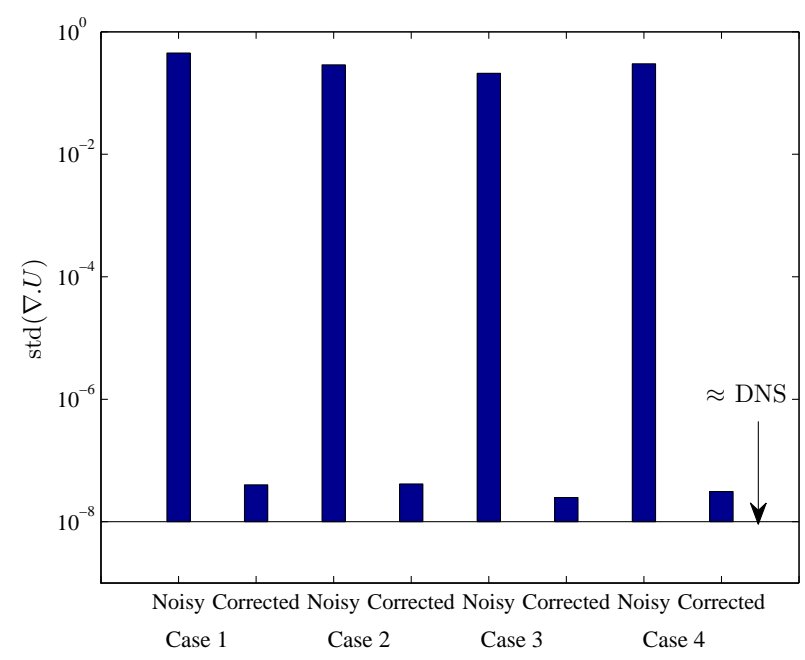

Fig. 12 Comparison of the standard deviation of the divergence error for the three cases considered in this study. For Case 1, noise is added based on equation (4). Similarly for Case 2, we use equation (5), and for Case 3 noise is added only to the streamwise velocity component based on equation (6) which considers bias in the error between the velocity components. Case 4 includes the influence of spatial averaging (bias errors towards the mean flow) commonly seen in 3D-3C measurements, where the noisy DNS velocity fields are filtered prior to correction by DCS. The black solid line indicates the mean divergence error from the original DNS velocity field.

The application of DCS to experimental data in a channel flow facility shows that a similar reduction in divergence to near zero is obtained, together with a closer proximity to DNS flow statistics computed at a matched Reynolds number. Analysis of the second and third invariants of the VGT shows that the experimental velocity field after correction by DCS is more representative of the measured velocity field when compared to previous studies performed on turbulent channel flows using DNS data [Blackburn et al., 1996]. It should be noted that the measurement quality is important for DCS to be successful. However as detailed in the experimental validation the uncertainty and noise level from a typical 3D-3C measurement is tolerable for DCS to work efficiently. In conclusion, although the verification done here employs a Tomo-PIV experimental data set, this technique can be applied to a wide variety of 3D-3C measurement techniques, including holographic PIV (HPIV) [Barnhart et al., 1994] and 3D particle tracking velocimetry (PTV) [Maas et al., 2004] to name a few.

Acknowledgements The authors wish to gratefully thank Prof. R.D. Moser for making the $R e_{\tau}=934$, DNS data available, and the financial support of the Australian Research Council. We would also like to thank Dr. Nicholas Hutchins for his help during the course of this study. 


\section{References}

R. J. Adrian and J. Westerweel. Particle Image Velcimetry. Cambridge University Press, 2011.

D. H. Barnhart, R. J. Adrian, and G. C. Papen. Phaseconjugate holographic system for high-resolution particle-image velocimetry. Appl. Opt., 33(30):7159$7170,1994$.

H. M. Blackburn, N. N. Mansour, and B. J. Cantwell. Topology of fine-scale motions in turbulent channel flow. J. Fluid Mech., 310:269-292, 1996.

H.H. Bruun. Hot-Wire Anemometry: Principles and Signal Analysis. Oxford University Press, 1995.

O. Buxton, S. Laizet, and B. Ganapathisubramani. The effects of resolution and noise on kinematic features of fine-scale turbulence. Exp. Fluids, 51:1417-1437, 2011.

M. S. Chong, A. E. Perry, and B. J. Cantwell. A general classification of three-dimensional flow fields. Physics of Fluids A: Fluid Dynamics, 2(5):765-777, 1990.

K. Christensen and R. J. Adrian. Measurement of instantaneous eulerian acceleration fields by particle image accelerometry: method and accuracy. Exp. Fluids, 33:759-769, 2002.

T. H. Clark. Measurement of Three-Dimensional Coherent Fluid Structure in High Reynolds Number Turbulent Boundary Layers. PhD thesis, Trinity Hall, Cambridge, 2012.

C. M. de Silva, R. Baidya, M. Khashehchi, and I. Marusic. Assessment of tomographic PIV in wall-bounded turbulence using direct numerical simulation data. Exp. Fluids, 52(2):425-440, 2012a.

C. M. de Silva, R. Baidya, and I. Marusic. Assesment of tomographic PIV using experimental and DNS data in turbulent channel flows. $16^{\text {th }}$ Int Symp on Applications of Laser Techniques to Fluid Mechanics, Lisbon, Portugal, 09-12 July, 2012b.

C. M. de Silva, R. Baidya, and I. Marusic. Enhancing Tomo-PIV reconstruction quality by reducing ghost particles. Meas. Sci. Tech., 24, 2013.

J. C. del Alamo, J. Jimenez, P. Zandonade, and R. D. Moser. Scaling of the energy spectra of turbulent channels. J Fluid Mech, 500:135-144, 2004.

G. E. Elsinga, F. Scarano, B. Wieneke, and B. W. van Oudheusden. Tomographic particle image velocimetry. Exp. Fluids, 41:933-947, 2006.

J M Foucaut and M Stanislas. Some considerations on the accuracy and frequency response of some derivative filters applied to particle image velocimetry vector fields. Meas. Sci. Tech., 13(7):1058, 2002.

B. Ganapathisubramani, E. K. Longmire, I. Marusic, and S. Pothos. Dual-plane piv technique to determine the complete velocity gradient tensor in a turbulent boundary layer. Exp. Fluids, 39:222-231, 2005.

J. C. Klewicki, J. A. Murray, and R. E. Falco. Vortical motion contributions to stress transport in turbulent boundary layers. Phy. Fluids, 6(1):277-286, 1994.

H. Maas, A. Gruen, and D. Papantoniou. Particle tracking velocimetry in three-dimensional flows. Exp. Fluids, 15:133-146, 2004.

J. P. Monty. Developments in smooth wall turbulent duct flows. PhD thesis, The University of Melbourne, 2005.

H.C. Ng. Experiments in smooth wall turbulent channel and pipe flows. $\mathrm{PhD}$ thesis, The University of Melbourne, 2011.

A. Ooi, J. Martin, J. Soria, and M. S. Chong. A study of the evolution and characteristics of the invariants of the velocity-gradient tensor in isotropic turbulence. J. Fluid Mech., 381:141-174, 1999.

S. R. Park and J. M. Wallace. The influence of instantaneous velocity gradients on turbulence properties measured with multi-sensor hot-wire probes. Exp. Fluids, 16:17-26, 1993. ISSN 0723-4864.

S. Tokgoz, G.E. Elsinga, R. Delfos, and J. Westerweel. Spatial resolution and dissipation rate estimation in taylor-couette flow for tomographic piv. Experiments in Fluids, 53(3):561-583, 2012.

P. Vukoslavcevic, J. M. Wallace, and J.-L. Balint. The velocity and vorticity vector fields of a turbulent boundary layer. part 1. simultaneous measurement by hot-wire anemometry. J. Fluid Mech., 228:25-51, 1991.

J. Westerweel and F. Scarano. Universal outlier detection for PIV data. Exp. Fluids, 39(6):1096-1100, 2005.

N. A. Worth, T. B. Nickels, and N. Swaminathan. A tomographic PIV resolution study based on homogeneous isotropic turbulence DNS data. Exp. Fluids, 49:637-656, 2010. 